\title{
Behaviour of scintillators under XUV free electron laser radiation
}

\author{
M. Kirm ${ }^{\mathrm{a}, *}$, V. Babin ${ }^{\mathrm{a}}$, E. Feldbach ${ }^{\mathrm{a}}$, S. Guizard ${ }^{\mathrm{b}}$, M. De Grazia ${ }^{\mathrm{c}}$, \\ V. Nagirnyi ${ }^{\mathrm{a}}$, A. Vasil'ev ${ }^{\mathrm{d}}$, S. Vielhauer ${ }^{\mathrm{a}}$ \\ ${ }^{a}$ Institute of Physics, University of Tartu, Riia 142, 51014 Tartu, Estonia \\ ${ }^{\mathrm{b}}$ Lab. des Solides Irradiés, CEA/DSM/DRECAM-CNRS Ecole Polytechnique, Palaiseau, France \\ ${ }^{\mathrm{c}} \mathrm{CEA} / \mathrm{DSM} / \mathrm{DRECAM} /$ Service des Photons, Atomes et Moleculés, Gif-sur-Yvette, France \\ ${ }^{\mathrm{d}}$ Physics Faculty, M.V. Lomonosov Moscow State University, 119992 Moscow, Russia
}

Available online 20 February 2008

\begin{abstract}
Free electron lasers (FEL) are new generation accelerator-based short wavelength light sources providing high pulse intensity and femtosecond pulse duration, which enable investigation of interaction of elementary excitations in solids under extreme conditions. Using the FLASH facility of HASYLAB at DESY (Hamburg, Germany), we investigated the response of different materials with scintillating properties based on intrinsic emissions to the 25.6 and $13.8 \mathrm{~nm}$ FEL radiation by means of time-resolved luminescence spectroscopy. FLASH delivered single pulses of $25 \mathrm{fs}$ duration having energy per pulse up to $30 \mu \mathrm{J}$ resulting in power densities of $\sim 10^{12} \mathrm{~W} / \mathrm{cm}^{2}$ on crystals. As a function of excitation density we observed the shortening of lifetime and non-exponential behaviour of emission decays in $\mathrm{CaWO}_{4}$, while the emission spectra recorded are comparable to those obtained at conventional excitation sources.

(C) 2007 Elsevier B.V. All rights reserved.
\end{abstract}

Keywords: Free electron laser; XUV radiation; Scintillators; Luminescence decay; Excitation density effects; $\mathrm{CaWO}_{4}$

Free electron lasers (FEL) are new generation accelerator-based short wavelength light sources with advanced properties (high pulse intensity, femtosecond pulse duration, monochromatic radiation, coherence, etc.), enabling new kinds of investigations in different scientific fields under extreme conditions. A pioneering test-experiment at TTF-1 facility in Hamburg had shown that interaction of $89 \mathrm{~nm}$ FEL radiation with scintillators leads to a remarkable shortening of the decay time and a drop in quantum yield of luminescence (e.g., in $\mathrm{BaF}_{2}$ and YAG:Ce) [1]. Analogous observation was reported for a number of inorganic scintillators in Ref. [2], where polychromatic hard X-ray synchrotron radiation was applied in experiments. It is obvious that, due to increasing peak power of new light sources, scintillator screens leave the region of linear response, in which they have been used so far to visualise intense radiation and particle beams. In the nonlinear regime effects arising from mutual interaction of elementary excitations can be investigated in more detail in

\footnotetext{
${ }^{*}$ Corresponding author. Fax: +3727383033 .

E-mail address: marco.kirm@ut.ee (M. Kirm).
}

comparison with conventional radiation sources as discussed in Ref. [3].

Using the BL1 of the FLASH facility of HASYLAB at DESY (Hamburg, Germany) [4], we investigated the response of different scintillation materials based on intrinsic or activator emission to 25.6 and $13.8 \mathrm{~nm}$ FEL radiation in single bunch mode with a pulse duration of $25 \mathrm{fs}$ and maximum energy of $30 \mu \mathrm{J}$ at $5 \mathrm{~Hz}$ repetition rate. By applying a $\mathrm{N}_{2}$ gas absorber, the incident beam could be attenuated up to two orders of magnitude from the estimated maximum value of $\sim 10^{12} \mathrm{~W} / \mathrm{cm}^{2}$. Luminescence spectra, typically averaged over 2000 FEL pulses, were recorded with a CCD camera mounted on the exit arm of the ARC SpectraPro308i imaging spectrograph. Emission spectra are presented as recorded without any correction to the sensitivity of detector and transmission of spectrograph. A XP2020Q PMT connected to a digital scope was used to record luminescence decays, through a set of bandpass filters selecting emission, for each single FEL shot. Analogously, the FEL pulse energy was measured for each shot by a gas monitor detector and stored, making possible to correlate this data with the respective decay curves later. 
Fig. 1 demonstrates the luminescence spectra recorded from a $\mathrm{CaWO}_{4}$ crystal at 300 and $8 \mathrm{~K}$, being in good agreement with literature data (see Ref. [5] and references therein). The luminescence has intrinsic origin due to radiative transitions within the oxyanionic complex, which form Frenkel type small radius self-trapped excitons in tungstates [6]. In the studied power density range, and for temperatures between 8 and $300 \mathrm{~K}$ no drastic changes in the shape of emission spectra were observed, except those naturally expected from a temperature change. Hence the nature of emission centres remains unaltered and the contribution of possible radiation damage, which can introduce new spectral features, is negligible. To clarify this point even more, we performed a special experiment, where sample was continuously irradiated and emission spectra were recorded as a function of time over longer period $(\sim 1 \mathrm{~h})$. Only the intensity fluctuations due to the SASE effect of FEL radiation were observed, but no decrease on average luminescence intensity was detected, which could be related directly to the radiation damage.

The dynamical behaviour of electronic excitations, which can be revealed via studies of luminescence decay kinetics is more sensitive to their mutual interaction. Recently, decay studies have been applied for the case of $\mathrm{CdWO}_{4}$ scintillator at room temperature excited by a HHG generated XUV photon-beam at the Saclay Laser-Matter Application Centre [7]. Fig. 2 presents the decays of intrinsic emission in $\mathrm{CaWO}_{4}$ at different temperatures and excitation densities. In the first few $\mu$ s all decays are strongly non-exponential, which is the primary result of a

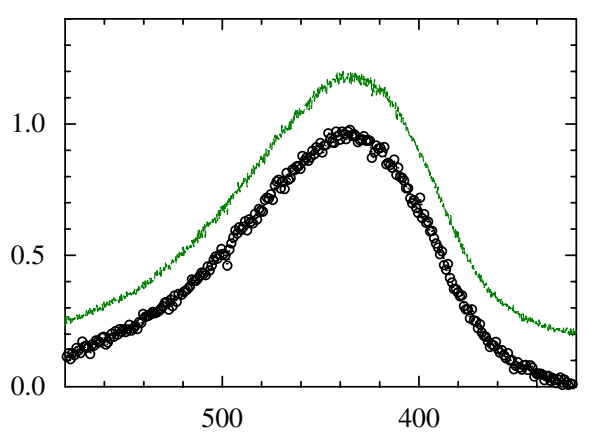

b

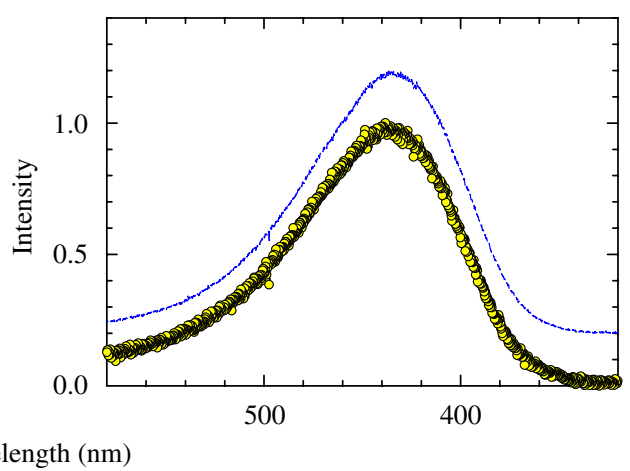

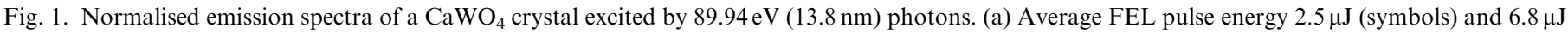

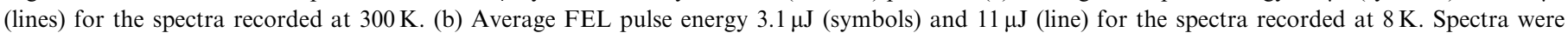
accumulated over 2000 FEL pulses and normalised at their maxima.

a

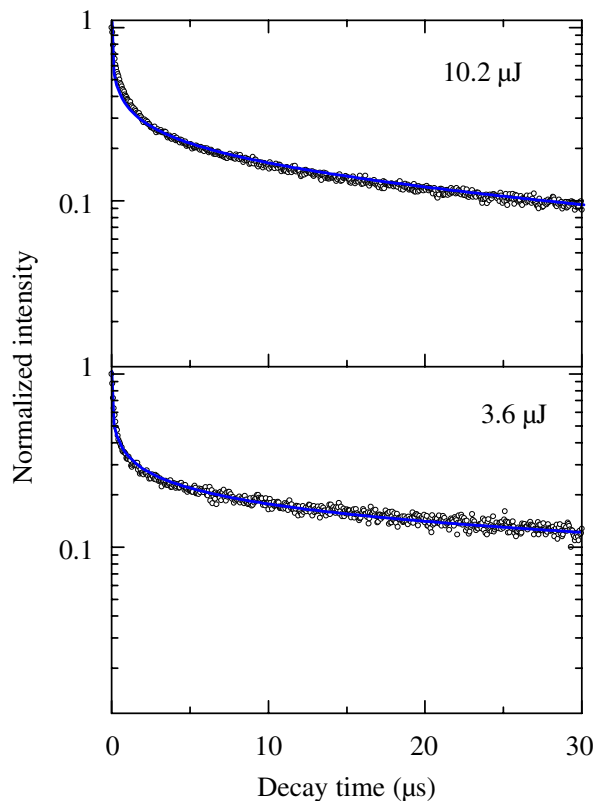

b

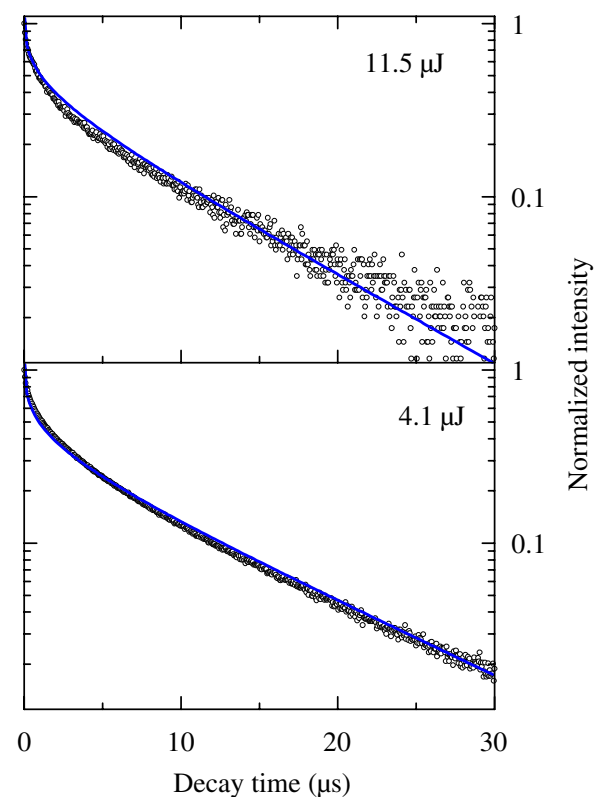

Fig. 2. Single-shot decay curves for the main emission of a $\mathrm{CaWO}_{4}$ crystal excited at $8 \mathrm{~K}$ (a) and $300 \mathrm{~K}$ (b) by $89.94 \mathrm{eV}$ (13.8 nm) photons. The pulse energies for each individual shot are noted on the plots. Symbols represent experimental data and lines are the results of fitting as described in the text. 
exciton-exciton interaction, whereas all electron-electron scattering processes occur on a timescale at least eightorders of magnitude faster than the lifetime of this luminescence centre. After this initial phase decays become considerably slower, and can be approximated by a single exponential function similar to low-density excitation using conventional sources. The decay time of this long component at room temperature is $8.5 \mu \mathrm{s}$, which agrees well with the lifetimes published earlier for $\mathrm{CaWO}_{4}$ [5]. At low temperatures, it possesses values of the order of $60 \mu \mathrm{s}$ (Fig. 2a) indicating the actual temperature at the surface of a $5 \mathrm{~mm}$ thick sample to be near $17 \mathrm{~K}$. It is important to note that in the range $4-50 \mathrm{~K}$ the decay time of $\mathrm{CaWO}_{4}$ changes exponentially with temperature, as shown under low-density photoexcitation [8]. Data analysis revealed also that there is a trend towards decreasing decay times of the long component with the increase of FEL pulse energy.

Assuming dipole-dipole interaction between closely spaced excitons and taking into account the spatial intensity distribution within the exciting light pulse such decays can be simulated by the theoretical approach based on kinetic equations describing $\mathrm{A}+\mathrm{A} \rightarrow \mathrm{A}$ interaction. $\mathrm{A}$ short description and a successful application in the quantitative analysis of non-exponential decays due to exciton-exciton interaction effects in $\mathrm{CdWO}_{4}$ at $300 \mathrm{~K}$ is found in Refs. [7,9]. In such a process, energy is released from one exciton due to its non-radiative decay and transferred to a neighbouring exciton. As a result this one will be ionised, leading to the remarkable deviation from the exponential decay during a short period after the exciting pulse (depending on the lifetime of excitons), when the concentration of excitations is still very high. The curves shown in Fig. 2 are modelled using this theoretical approach. Quantitative results on the behaviour of different scintillators and luminescent materials under powerful FEL excitation will be presented in more detail elsewhere [10].

The present study demonstrates, using intrinsic emission of $\mathrm{CaWO}_{4}$ as an example, that the shortening of lifetime and non-exponential behaviour of emission decays occurs as a function of excitation density. The emission spectra recorded at power densities $\sim 10^{12} \mathrm{~W} / \mathrm{cm}^{2}$ are comparable to those obtained at conventional excitation sources. Also surface damage was found on the post-experiment inspection of the $\mathrm{CaWO}_{4}$ crystal. In future, high power XUV FEL allows systematic studies of dynamical processes in a regime of strongly interacting excitations in solids, which cause effects like the non-proportionality of scintillator response and others. The very short femtosecond pulse lengths make FEL also very attractive for pump-probe experiments, which can look directly into relaxation dynamics in wide gap solids.

Support by the Estonian Science Foundation (Grants 6538 and 0548J), and by the European Community Research Infrastructure Action in the FP6 Program of the EU (Contract RII3-CT-2004-506008 (IA-SFS)) and the HRM Activity of the EU, Marie Curie Action XRTA RTN are gratefully acknowledged.

\section{References}

[1] M. Kirm, A. Andrejczuk, J. Krzywinski, R. Sobierajski, Phys. Stat. Sol. (c) 2 (2005) 649.

[2] V.A. Pustovarov, A.L. Krymov, E.I. Zinin, Nucl. Instrum. Methods A 359 (1995) 336.

[3] M. Kirm, V. Babin, E. Feldbach, V. Nagirnyi, S. Vielhauer, B. Carré, S. Guizard, M. De Grazia, H. Merdji, A.N. Belsky, N. Feodorov, P. Martin, Phys. Stat. Sol. (c) 4 (2007) 870.

[4] V. Ayvazyan, N. Baboi, J. Bähr, et al., Eur. Phys. J. D 37 (2006) 297.

[5] V.B. Mikhailik, H. Kraus, D. Wahl, M. Itoh, M. Koike, I.K. Bailiff, Phys. Rev. B 69 (2004) 205110.

[6] V. Nagirnyi, P. Dorenbos, E. Feldbach, L. Jönsson, M. Kerikmäe, M. Kirm, E. van der Kolk, A. Kotlov, H. Kraus, A. Lushchik, V. Mikhailik, R. Sarakvasha, A. Watterich, in: Proceedings of the Eighth International Conference on Inorganic Scintillators and Their Use in Scientific and Industrial Applications, Alushta, Ukraine, 2005, pp. 36-39 (Natl. Acad. Sci., Kharkov, 2006).

[7] A. Belsky, N. Fedorov, P. Martin, S. Guizard, G. Geoffroy, J. Gaudin, G. Petite, M. De Grazia, H. Merdji, B. Carré, M. Kirm, V. Nagirnyi, E. Feldbach, A. Vasil'ev, J. Phys. IV 138 (2006) 155.

[8] V. Nagirnyi, A. Kotlov, L. Jönsson, M. Kirm, A. Lushchik, Nucl. Instrum. Methods A 537 (2005) 61.

[9] N. Fedorov, V. Nagirnyi, A. Vasil'ev, A. Belsky, B. Carré, E. Feldbach, J. Gaudin, G. Geoffroy, S. Guizard, M. De Grazia, M. Kirm, P. Martin, H. Merdji, G. Petite, J. Phys. IV 138 (2006) 251.

[10] S. Vielhauer, V. Babin, M. De Grazia, E. Feldbach, M. Kirm, V. Nagirnyi, A. Vasil'ev, Phys. Solid State (2008), in press. 